\title{
The contents of perceptual hypotheses: Evidence from rapid resumption of interrupted visual search
}

\author{
Justin A. Jungé, Timothy F. Brady, And Marvin M. Chun \\ Yale University, New Haven, Connecticut
}

\begin{abstract}
Observers can resume a previously interrupted visual search trial significantly more quickly than they can start a new search trial (Lleras, Rensink, \& Enns, 2005). This rapid resumption of search is possible because evidence accumulated during the previous exposure, a perceptual hypothesis, can carry over to a subsequent presentation. We present four interrupted visual search experiments in which the content of the perceptual hypotheses used during visual search trials was characterized. These experiments suggest that prior to explicit target identification, observers have accumulated evidence about the locations, but not the identities, of local, task-relevant distractors, as well as preliminary evidence for the identity of the target. Our results characterize the content of perceptual search hypotheses and highlight the utility of interrupted search for studying online search processing prior to target identification.
\end{abstract}

Locating objects in the local environment is essential for successful navigation in a complex world, and visual search can operate across a wide variety of environmental conditions and over a remarkable repertoire of useful feature combinations. The central functionality of visual search has attracted considerable empirical investigation and theoretical consideration over the past several decades. Search experiments typically ask participants to locate and respond to a predefined target object in a field of distractors. Designs of this type are well suited for exploring the nature of attentional selection and the time course of processing a variety of simple and complex stimuli. Much of what is known today about visual search has been deduced from search slopes. By adding more distractors to a display containing a single target and observing the corresponding increase in average response time (RT), it is possible to infer the average amount of processing time for each additional distractor. Prominent early theories of attention and visual search made extensive use of evidence from search slopes (e.g., Duncan \& Humphreys, 1989; Treisman \& Gelade, 1980). However, there remain important questions about search processing that may not be readily addressed using search slopes alone. All processing prior to target identification and response gets lumped under the same RT in traditional studies of visual search, but new methods and analyses can provide a window into search processing prior to target detection. The phenomenon of rapid resumption (Lleras, Rensink, \& Enns, 2005) - to be discussed at length below-may provide one such source of converging evidence and additional inference.

Visual search requires both attentional selection and several types of memory (Kristjánsson, 2000; Peterson, Kramer, Wang, Irwin, \& McCarley, 2001; Shore \& Klein, 2000; Woodman \& Chun, 2006). It has been suggested that in order to perform typical search tasks, a target template must be held in working memory (Duncan \& Humphreys, 1989). In fact, top-down influences on search are essential in most models of visual search. This has led researchers to investigate the possibility of shared resources between visual search and working memory tasks, using dual-task designs. Loading executive working memory impairs visual search efficiency (Han \& Kim, 2004), as does loading spatial working memory (Oh \& Kim, 2004; Woodman \& Luck, 2004). However, actively remembering certain simple feature details, such as color patches, does not seem to affect search slopes (Woodman, Vogel, \& Luck, 2001). There is reason to think that independently of resource sharing, memory impacts search in the form of accumulated (preliminary) evidence within a given trial. At a minimum, extracting the identity of the target must cross a threshold of recognition, and the processing prior to crossing this threshold qualifies as preliminary evidence accumulated. Rapid resumption provides a new way to study the information that accumulates about targets and distractors prior to target detection.

The phenomenon of rapid resumption was discovered using an interrupted visual search task. Each visual search

J. A. Jungé, justinjunge@gmail.com 
array contained one target, the letter $T$, embedded in a field of distractor $L$ s. Each search array was presented to participants in successive flashes: on for $100 \mathrm{msec}$, off for $900 \mathrm{msec}$, in a repeated loop (On-Off-On-Off . . .) until they located the target $T$ and responded to it. The design yielded two important patterns. First, there was a startup cost: The participants almost never responded within $500 \mathrm{msec}$ after the onset of the initial presentation. Second, there was a signature of accumulated memory from one presentation to the next, evident in the rapid responses that often occurred (about $30 \%-50 \%$ of the responses) less than $500 \mathrm{msec}$ after the onset of any presentation subsequent to the first. Lleras et al. (2005) referred to the useful accumulated information that facilitates rapid responses as perceptual hypotheses, building from a theory of reentrant processing (e.g., Di Lollo, Enns, \& Rensink, 2000).

Theories of reentrance propose that representations formed and maintained in high-level visual areas are checked against the corresponding activity in lower level visual areas, which, in turn, generally corresponds to the currently viewed stimuli. When the top-down signal matches the bottom-up activity, that content becomes available for report. Under this framework, visual search is accomplished using iterative loops of processing, with stages of extraction and confirmation. Information is implicit while first being extracted, in the perceptual hypothesis formation stage, and it becomes explicit once a hypothesis is confirmed, sometimes resulting in target identification and response. If a viewed item is replaced before a perceptual hypothesis can be confirmed, the content of that hypothesis is discarded (consistent with the multiple-drafts theory of conscious awareness; Dennett, 1991). This could help explain how trailing mask stimuli impair target detection, even when masks do not spatially overlap with the targets (e.g., object substitution masking). A reentrant theory of masking proposes that targets may escape conscious report because of a certain type of perceptual mismatch from one moment to the next, close enough to warrant comparison, but different enough to induce the disconfirmation of a perceptual hypothesis (Di Lollo et al., 2000). However, if a perceptual search hypothesis containing a target item is formed prior to removal of an entire search display, then when the display reappears, the hypothesis can be tested immediately, resulting in rapid responses. The interrupted search design makes it possible to selectively disrupt a processing loop and observe the consequences. We emphasize again that, in this article, perceptual hypothesis refers to implicit stages of processing prior to confirmation and awareness of the target. Thus, the term should be clearly distinguished from explicit hypotheses (e.g., "I think the target is in the top right corner").

Conceiving of search processing in terms of perceptual hypotheses raises interesting questions about the content of such hypotheses: Do perceptual hypotheses contain information about the target alone, the entire search display, or a subset of items? What is the spatial and featural resolution of perceptual hypotheses? And, are they formed exclusively over task-relevant items? In order to address precisely these questions, we introduced changes between successive displays to measure which changes would disrupt rapid resumption. Implicit perceptual hypotheses need to be tested against actual displays. Thus, any change made to a display after implicit hypothesis formation could potentially interfere with the confirmation stage, reducing the occurrence of rapid resumption. By making slight changes to a display (such as altering the location or orientation of a controlled subset of items while the display is out of sight), it becomes possible to observe any corresponding decrement in rapid resumption and infer that the changed features were part of the implicit perceptual hypotheses.

\section{EXPERIMENT 1}

\section{The Spatial Extent of Perceptual Hypotheses}

Interrupted search design and rapid resumption provide a sensitive measure for looking at the spatial extent of concurrent processing. Do perceptual hypotheses contain only one item, several items, or entire search displays? To test this, we changed the locations of several distractor items between presentations of a search array, dissecting perceptual hypothesis formation and test. By varying the distance between moved distractor items and the target, it was possible to investigate whether all the changes would affect rapid resumption equally.

\section{Method}

Participants. Twenty-six students participated for a monetary payment or partial course credit. All the participants were naive as to the purpose of the experiment and had normal or corrected-tonormal acuity.

Apparatus. All the experiments were programmed in MATLAB, using the Psychophysics Toolbox extensions (Brainard, 1997), and were conducted on a Macintosh computer with a 17-in. screen. The participants viewed the displays without restraint from approximately $57 \mathrm{~cm}$.

Stimuli. The participants performed a search task, locating a rotated target $T$ among rotated distractor $L$ s and responding to the orientation of the target $T$. The two possible target $T \mathrm{~s}$ (subtending $1.6^{\circ}$ ) were rotated by either $90^{\circ}$ or $270^{\circ}$, and exactly one was present on every trial. The distractor $L$ s were randomly rotated $\left(0^{\circ}, 90^{\circ}, 180^{\circ}\right.$, or $270^{\circ}$ ). Each $L$ also subtended approximately $1.6^{\circ}$, and the point of contact between the longer and shorter bars was offset toward center by $0.15^{\circ}$, to increase search difficulty. All the search items were white, presented on a uniform gray background.

Each search array contained 16 items, 4 in each quadrant of the screen. Displays were constructed using 36 evenly distributed potential locations, with 9 in each quadrant. Each item was placed into 1 of these locations and was then randomly jittered by $0.0^{\circ}-0.5^{\circ}$. The target location was chosen randomly from a subset of 28 locations, excluding the 4 locations surrounding the center, as well as 1 location in each corner of the display.

Design and Procedure. The participants were instructed to respond as quickly and accurately as possible to a target $T$ by pressing one of two keys to indicate its orientation. On each trial, the search display would appear for $500 \mathrm{msec}(\mathrm{on})$, followed by a blank screen for 1,600 msec (off), and this on-off cycle would repeat until a response was made (one complete on-off cycle will subsequently be referred to as an epoch). These parameters were set to correspond to Experiment 3B in Lleras et al. (2005) - the condition in which they observed the greatest proportion of rapid resumption. All the participants initially completed $10-20$ practice trials, until they reported being comfortable with the task, and their performance on these trials was not recorded. 
There were three conditions with an equal number of trials in each, and these conditions were randomly intermixed across trials throughout the experiment. The no-change condition (one third of all the trials) served as a baseline; displays were repeated across epochs. In the near change and distant change conditions, four of the distractor $L$ s moved in between search presentations (during the off part of the trials) - two distractor items in two diagonally opposed quadrants. These moves were made relative to target location. On half of the change trials, one of the two changed quadrants also contained the target (near change), and on the other half of the change trials, the changed quadrants did not contain the target (distant change). The target never moved, and all the moved items remained within the same quadrant of the display as that in which they had started. Apart from the described location changes, the search displays were unchanged between presentations. The participants completed seven blocks of 48 trials, with a chance for a brief rest between blocks.

Quantifying rapid resumption. In this and all the subsequent experiments, we follow Lleras et al. (2005) in operationally defining rapid resumption as responses occurring under $500 \mathrm{msec}$ after display reappearance. Although any strict cutoff runs the risk of generating a spurious dichotomy, there are pragmatic and theoretical reasons to employ the chosen 500-msec cutoff. One reason is that it effectively distinguishes between observed performance for the initial stimulus presentation (Epoch 1) and that for all subsequent epochs (Lleras et al., 2005). Without assigning some cutoff, this robust pattern would go undetected. But why $500 \mathrm{msec}$ ? A striking feature of the cumulative response distribution, for all epochs subsequent to the initial presentation, is a bimodal distribution with a trough centered at approximately $500 \mathrm{msec}$ (although see the General Discussion section regarding exceptions) and peaks approximately $100-200 \mathrm{msec}$ before and after. This distribution is suggestive of a process operating in cycles with relatively stable temporal dynamics. A theoretical account that pins down the underlying nature of the bimodal response distribution could potentially justify the 500msec threshold or revise it. At present, this threshold is employed pragmatically, following prior convention.

\section{Results}

Data from 3 participants were excluded from all subsequent analyses due to excessively high error rates (over three standard deviations above the mean, applied iteratively), and the error rates for the remaining participants were acceptably low, with a mean of $3.3 \%$. All analyses were performed exclusively over RTs for correct trials. In line with Lleras et al. (2005), we quantified rapid resumption as responses made within $500 \mathrm{msec}$ after reexposure to an incomplete search trial and focused on Epochs 2-6. Exemplifying the reliability of this 500 -msec cutoff, fewer than $2 \%$ of all the responses made during the first exposure (Epoch 1) fell under it, whereas more than $30 \%$ of all the responses in Epochs 2-6 were in this range.

The mean proportion of responses under $500 \mathrm{msec}$ in each condition was as follows: no change, $35.7 \%$; near change, $32.0 \%$; distant change, $35.6 \%$. Near change trials exhibited significantly less rapid resumption than did no-change trials $[t(22)=2.16, p=.04]$, as well as significantly less than did distant change trials $[t(22)=2.43$, $p=.02]$. However, no-change and distant change trials did not significantly differ $[t(22)=0.07, p=.95]$.

Figure 1 depicts the proportion of responses in Epochs 2-6 for each condition, at intervals of $100 \mathrm{msec}$, following display onset for each epoch. The first apparent trend is a bimodal distribution with a higher first peak at about $400 \mathrm{msec}$, and a lower second peak around $700 \mathrm{msec}$. Consistent with fewer rapid responses in the near change condition, there was a corresponding rebound in this condition at the 700-msec peak (however, differences in this 600- to 700-msec bin were not significant).

\section{Discussion}

The content of perceptual hypotheses can be probed when changes made to displays between successive viewings reduce rapid resumption, due to a mismatch between perceptual hypotheses and the actual search displays. The results of Experiment 1 demonstrate that perceptual search hypotheses are sensitive to the locations of distractors relatively near the target, but not distractor locations distant from the target. These effects suggest a scope of approximately three to four item locations, the number

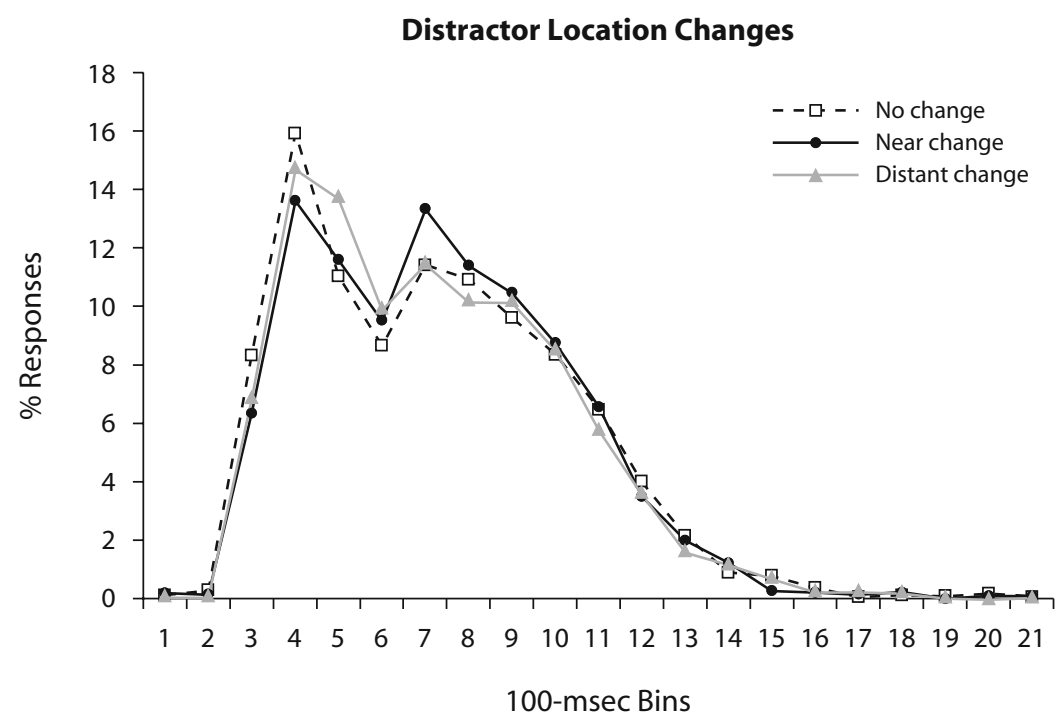

Figure 1. Average response times over the course of 2,100-msec epochs, for Epochs 2-6, grouped into 100-msec bins. 
of search items present in each quadrant of this experiment, although additional investigation should determine whether these effects depend specifically on the number of items or the spatial proximity of those items to a target. Distractor locations are fairly low resolution, are accessible in both low and high spatial frequencies, and may be encoded without feature details. Locations provide a consistent spatial context that may aid localization of a target (Brady \& Chun, 2007; Chun \& Jiang, 1998; Jiang, Olson, $\&$ Chun, 2000). For this reason, locations may be especially useful for matching a formed perceptual hypothesis to the currently available visual input. Featural details such as item identity and directional orientation require greater resolution to represent, and in the present study, they are only task-relevant features of a target. Holding onto evidence about distractor feature details may be inefficient when these details are not task relevant.

\section{EXPERIMENT 2A Distractor Resolution}

The visual system has numerous capacity limitations, and during visual search certain information will be processed, and other information must be discarded. Thus, as was noted above, it may not be efficient or feasible for perceptual hypotheses to maintain high-resolution details about distractors (e.g., Becker \& Pashler, 2002; see also Rauschenberger \& Yantis, 2006, for a discussion of efficiency in visual search). For example, in the present search experiments, as soon as the orientation of a distractor has been recognized, that identity provides no utility for target detection and can effectively be discarded. We tested whether perceptual hypotheses contain orientation information about distractors.

\section{Method}

The method for Experiment 2A was identical to that for Experiment 1 , except that changes were made to the orientations of distractor items (a rotation of $90^{\circ}, 180^{\circ}$, or $270^{\circ}$ ), while always holding their locations constant. We tested 12 new participants, also naive as to the purpose of the experiment.

\section{Results}

One participant was excluded from all subsequent analyses for excessive errors (26.4\%), and error rates in other participants were acceptably low, with a mean of $4.7 \%$. Despite making changes to an equal number of items as in Experiment 1, there was no sign of disruption from the distractor orientation changes in Experiment 2A. The rate of rapid resumption by condition was: no change, $28.6 \%$; near change, $27.8 \%$; and distant change, $27.7 \%$. These rates of rapid resumption were not significantly different from each other [no change vs. near change, $t(11)=0.77, p=$ .46 ; no change vs. distant change, $t(11)=0.65, p=.53$; near change vs. distant change, $t(11)=0.73, p=.94]$.

\section{EXPERIMENT 2B Target Resolution}

The report attribute of a target in all of our present experiments is the directional orientation of the target $T$. We manipulated this variable to explore the featural resolution of targets in perceptual hypotheses. Experiment 2A demonstrated that perceptual search hypotheses are not sensitive to the directional orientation of distractors - at least, in a search task in which such a detail did not provide any benefit. However, it remains an open question whether feature details such as directional orientation can be included in implicit perceptual hypotheses at all. The alternative would be that search hypotheses contain merely location information similar to saliency maps (e.g., Wolfe, 1994). Such a model would direct attention to a location, without providing any expectation as to what is at that location. We tested whether perceptual hypotheses contain preliminary information about the orientation of the target by investigating whether changes to the target orientation would disrupt rapid resumption.

\section{Method}

Twelve new participants from the same population were tested. The method was identical to that in Experiment 2A, with the exception of the design details described below.

Design. There were only two conditions, no change and target change, and these trials were randomly intermixed throughout the blocks. On target change trials, the target switched orientation between presentations, as did one distractor in each of the other quadrants. The target always alternated between the two possible orientations, but the distractors changed randomly to any one of the three orientations different from the previous exposure. This equated the overall number of items changed to that in Experiment 2A, while manipulating the critical report attribute of the target. A preceding pilot run indicated that naive participants eventually noticed some target changes and broke from the experiment to inquire about the changes. Consequently, all the participants in this condition were informed that some items, including targets, could potentially change between presentations (epochs).

\section{Results}

Error rates were acceptably low at 3.2\%. In Epochs 2-6, rapid resumption in the no-change condition was at $30.4 \%$, and in the target change condition it dropped to $23.7 \%$. These rates were significantly different $[t(11)=4.01$, $p<.01]$, showing that changing the target interfered with rapid resumption (see Figure 2).

\section{Discussion}

Experiments 2A and 2B demonstrated that although it is possible to hold the orientation identity of at least one search item (e.g., the target) in a perceptual hypothesis, this level of resolution is evidently not used for distractors. Distractor features may be unlikely to provide additional utility for matching a hypothesis to a search array. As was shown in Experiment 1, distractor spatial location information is partially encoded in perceptual hypotheses, since spatial information provides contextual cues to the target location (Chun \& Jiang, 1998; Jiang et al., 2000). Details about distractor identity may be discarded due to capacity limits. However, even if resources were available, such details might not be encoded, because they did not provide any additional utility. From these considerations, sparse perceptual hypotheses appear to be both efficient and effective.

Experiments $2 \mathrm{~A}$ and $2 \mathrm{~B}$ were designed to compare distractor and target detail, and a very similar design has re- 


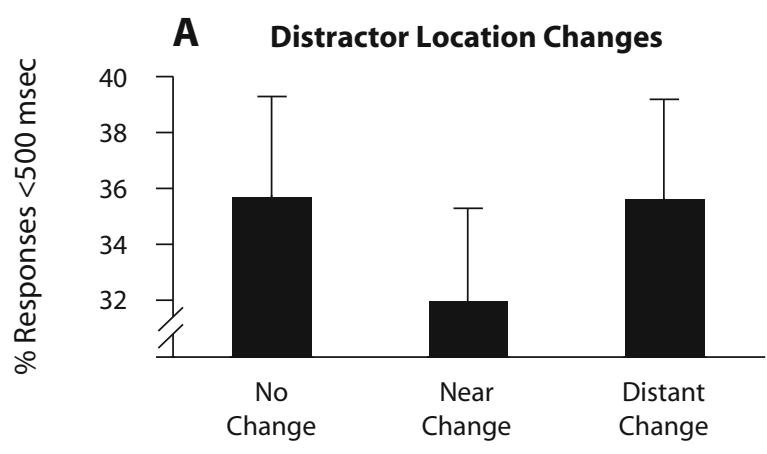

B Distractor Orientation Changes
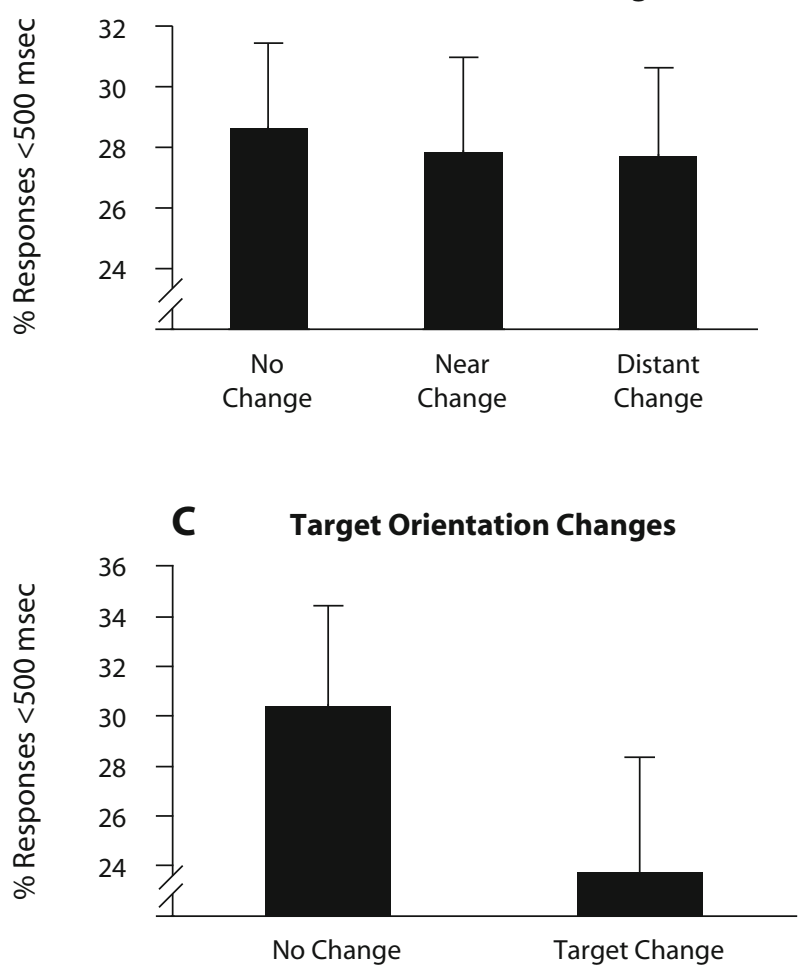

Figure 2. Mean number of rapid responses in each experimental condition during Epochs 2-6, comparing the no-change condition with select item changes. (A) Several distractor locations were changed in between epochs, either near the target or distant from it. (B) Several distractor orientations were changed in between epochs, either near the target or distant from it. (C) The target orientation was changed in between epochs.

cently been employed by Lleras, Rensink, and Enns (2007) to study the target specificity of rapid resumption. In a series of experiments, they found that changing all distractor locations between search presentations (between epochs) but leaving the target alone left rapid resumption relatively intact. However, changing the report attribute of the target significantly interfered with rapid resumption. Their experiments employed 100-msec on and 900-msec off epochs, and this may have limited the resolution of perceptual hypotheses. It has been reported that explicit visual working memory fills to capacity within approximately $500 \mathrm{msec}$ (Alvarez \& Cavanagh, 2004; Vogel, Woodman, \& Luck,
2006), but the capacity limit of implicit visual memory is difficult to specify (Jiang, Song, \& Rigas, 2005). Consistent with our results, Lleras et al. (2007, Experiment 4) found that changing the report attribute of a target (the color of a $\operatorname{target} T$ ) between presentation epochs interfered with rapid resumption. However, the interference observed was much greater in their study than in ours, entirely eliminating rapid resumption when responses occurred immediately after a change epoch. Their design employed within-trial variability, with changes occurring unpredictably between $50 \%$ of successive presentations (epochs). Our design employed between-trial variability, with changes occurring unpredictably in $50 \%$ of trials, but within these change trials the target report attribute (its orientation) alternated every successive presentation. Such consistent within-trial alternation could, in principle, allow preliminary target evidence to prime an opposite response or could help resolve response conflict. Both possibilities require further investigation.

Lleras et al. (2005) proposed that after a perceptual hypothesis containing a target has been generated, testing it is similar to having only a single item, the target, on the screen. Supporting this position, Lleras et al. (2007) observed rapid resumption even when all distractor locations changed between epochs (a stronger manipulation, consistent with our Experiment 1). Similarly, we observed rapid resumption in all our experimental conditions. However, we found that rapid resumption is significantly reduced by manipulations such as changes to local spatial context. Such a reduction was also apparent in Lleras et al. (2007, Experiment 2) but was not submitted to statistical analyses. In sum, rapid resumption is driven primarily by target location and identity information in perceptual hypotheses, although some local distractor context is encoded as well to facilitate search.

The scope of perceptual search hypotheses appears to be limited to the spatial location of distractor items near the target and includes some location and featural details of the target. Resource limitations can create bottlenecks where sparse representations are exceedingly useful. Having observed perceptual hypotheses consistent with sparse representations in Experiments 1, 2A, and 2B, we extended the present investigation to another variety of selectivity that may influence interrupted and resumed perception. One way to filter information during visual search is to apply a criterion that excludes unlikely target candidates. Forming perceptual hypotheses exclusively over task-relevant items could support efficient processing across multiple temporally distinct samples from the environment. Considering sharp visual capacity limits and the dynamic nature of scene perception, the possibility of selectively carrying information across samples could be efficient and informative. Experiment 3 investigated the strategic feature selectivity of perceptual search hypotheses.

\section{EXPERIMENT 3 Attentional Set and Task Relevance}

It is possible to filter the input to visual awareness on the basis of certain simple features, such as luminance, color, size, orientation, and motion (see, e.g., Egeth, Jonides, 
\& Wall, 1972; Egeth, Virzi, \& Garbart, 1984; Treisman \& Gelade, 1980; Wolfe, Butcher, Lee, \& Hyle, 2003). Feature-based filtering (i.e., attentional set) could potentially be implemented by top-down reentrant signaling, selectively amplifying a specified type of feature input from low-level feature maps. Selective attention constrains the type of low-level content included in perceptual hypotheses, and perceptual hypotheses should contain only items that share reliable surface features with a known type of target. Here, we tested this possibility using a fixed target luminance and two types of distractors, half the same luminance as the target and half different. If perceptual hypotheses are restricted by attentional set, changing the location of distractors between presentation epochs should influence rapid resumption only when those distractors fall within the attentional set of the target ${ }^{1}$ (and are spatially proximate to the target; see Experiment 1).

\section{Method}

We tested 12 new participants from the same population. The method was identical to that in Experiment 1, with the exception of the design details described below.

Stimuli. Each of the $T$-among- $L$ s search arrays for this experiment was composed of 12 black items and 12 white items. For half of the participants, throughout the entire experiment, the target (a rotated $T$ ) was always 1 of the 12 black items, and for the other half of the participants, the target was always 1 of the 12 white items. The 23 distractor items were all $L$ s rotated $0^{\circ}, 90^{\circ}, 180^{\circ}$, or $270^{\circ}$, as in the previous experiments.

Search arrays contained 64 evenly distributed potential locations, with 16 possible locations in each quadrant. Each quadrant contained three black items and three white items. The target was never placed in the 4 locations touching the center of the screen or in the corner locations.

Design and Procedure. The participants completed nine blocks of 40 trials, with a chance for a brief rest between blocks. Prior to beginning, each participant was told whether he or she would be searching for black or white targets throughout the experiment. An instruction screen displaying the two possible target orientations and their corresponding response keys was presented at the start of each block, with the target displayed with the luminance that it would have for the entire experimental session for that participant.
This experiment included a no-change condition, as well as two conditions in which select distractor items were moved (changed location between presentation epochs). In the attended condition, task-relevant items, having the same luminance as the target, were moved. In the unattended condition, task-irrelevant items, not having the same luminance as the target, were moved. Both of these conditions were also divided into near target moves and distant target moves, applying the same display quadrant manipulations as those in Experiments 1 and 2A. In near move trials, two distractor items in the same quadrant as the target were moved, along with two distractor items in the diagonally opposed quadrant. In distant move trials, two distractor items in each of two diagonally opposed quadrants (neither of which contained the target) changed location between presentations. All the moved items remained in their originating quadrants.

\section{Results}

Error rates were acceptably low for all the participants, with a mean of $3.8 \%$. The no-change trials were used as a baseline for each participant, such that the proportion of rapid resumption in the other conditions was considered relative to no change. We submitted these normalized data (differences in rapid resumption, as compared with no change) to a $2 \times 2$ repeated measures ANOVA, comparing task relevance (attended vs. unattended) and distance (near vs. distant, relative to the target) of the moved distractors. The interaction between these factors was not significant $[F(1,11)=1.59, p=.23]$. There was a significant main effect of task relevance $[F(1,11)=20.54$, $p<.01]$, but not of distance $[F(1,11)=0.94, p=.35]$ (see Figure 3).

The raw data were also subjected to planned comparisons to confirm that moving (attended) items in the task-relevant luminance interfered with rapid resumption, as compared with moving (unattended) items in the task-irrelevant luminance and no-change conditions. The attended change trials showed significantly less rapid resumption than did the unattended change trials $[t(11)=$ $4.53, p<.01]$ and marginally significantly less resumption than did the no-change trials $[t(11)=2.17, p=.05]$. This difference was apparently driven by the attended-near

\section{Task Relevance of Distractor Location Changes}

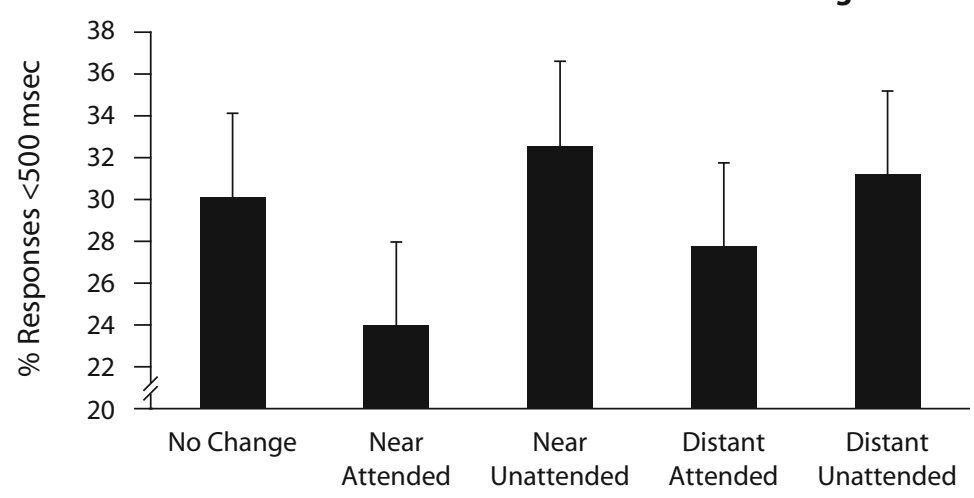

Figure 3. Mean number of rapid responses in each experimental condition during Epochs 2-6, comparing the no-change condition with select item changes. The task relevance of changed items was manipulated, along with distance from the target. Distractors that changed locations were either the same luminance as the target or a different luminance, and near the target or distant from it. 
trials, which differed significantly from no-change trials $[t(11)=2.82, p=.02]$, whereas the attended-distant trials did not $[t(11)=0.93, p=.37]$.

\section{Discussion}

The results of Experiment 3 confirm that task relevance modulates the effective content of perceptual hypotheses. Changing the locations of distractors that were the same luminance as the target interfered with rapid resumption, whereas moving distractors of a different luminance did not. Such selectivity, in conjunction with the location-only details extracted from nearby task-relevant distractors, suggests that perceptual hypotheses are limited in scope and encode minimal information required for a task. Although such limitations are a prominent feature of within-trial perceptual hypotheses, it may be possible to store the output of confirmed perceptual hypotheses in long-term memory to benefit future trials. The long-term storage of perceptual hypotheses will be considered in a subsequent discussion of implicit learning and priming during visual search.

\section{GENERAL DISCUSSION}

In the present experiments, we investigated the content of perceptual hypotheses during visual search, using the phenomenon of rapid resumption. Our results suggest that perceptual hypotheses are formed over a spatially local subset of up to three to four task-relevant items. These hypotheses focus on target identity and also include information about the locations of distractor items, but not their orientations (see Figure 2). Our results are consistent with Rensink's (2000) coherence theory, which proposes that attentional resources are limited to a single high-resolution item and sparse details about several neighboring items.

We should note that all positive results - effectively disrupting rapid resumption-are evidence for the accumulation of the corresponding information in perceptual hypotheses. However, failure to disrupt rapid resumption (as in the cases of distractor orientation changes and taskirrelevant distractor location changes) does not exclude the possibility that a more sensitive measure might find that these details accumulated in memory as well.

\section{Refining Rapid Resumption}

The phenomenon of rapid resumption was only recently discovered, and many open questions remain to be explored, several of which are directly relevant to the experiments reported here. For example, in all three experiments, we observed very large individual differences in base rates of rapid resumption (spanning $4 \%-81 \%$ at the extremes), using the fixed cutoff of $500 \mathrm{msec}$. These differences suggest a range of search and response strategies and make it quite difficult to compare absolute rates of rapid resumption between experiments. Nevertheless, within-participant variability between conditions was systematic and reliable, even in participants with very different base rates of rapid resumption.

Other factors likely to impact base rates of rapid resumption are the timing and regularity with which displays cycle on and off and, specifically, the predictability of search display reappearance. Testing implicit perceptual hypotheses may require a degree of explicit expectation regarding the likelihood that a hypothesis will be relevant to the upcoming search array (Lleras, Rensink, \& Enns, 2004). In the context of the present experiments, it is possible that explicitly noticing changes in between epochs influenced base rates of rapid resumption. However, as was noted above, determining effects on base rates of rapid resumption may require within-participants manipulations.

An additional consideration for interpreting rapid resumption is the cutoff used to define responses as rapid. The original reports of rapid resumption (Lleras et al., 2004, 2005) defended a 500-msec cutoff as optimally distinguishing performance on the first epoch and subsequent epochs. However, manipulating task difficulty could have produced an earlier or later cutoff. A converging point of consideration was the bimodal response distribution in epochs subsequent to the first epoch, with a local minima centered at approximately $500 \mathrm{msec}$. This minima appears to shift to slightly later when exposure times are increased (e.g., in our Experiment 1, the minima appears closer to $600 \mathrm{msec}$ ). Both distinguishing Epoch 1 from subsequent epochs, and locating the minima in the bimodal response distribution of later epochs implicate approximately $500 \mathrm{msec}$ as a good cutoff. However, future investigation should closely examine the flexibility of these factors and the possible validity of alternative thresholds.

\section{Reentrance and Visual Information}

Visual information undergoes a substantial sequence of transformations between retinal stimulation and awareness. A fundamental goal of perception research is to characterize landmarks in the processing sequence of visual input, such as the point(s) at which implicit content becomes explicit and response selection occurs. Reentrance provides a model of the passage from implicit to explicit visual processing (e.g., Di Lollo et al., 2000; Lleras et al., 2005). There are three prominent stages in such a theory: (1) Visual input travels from low-level processing of featural details up to high-level processing of object identity; (2) the results of high-level analyses are then compared with low-level activity; and (3) this double-checked output is passed into awareness for response selection.

Under the reentrant model, Lleras et al. (2005) stated that the content of perceptual hypotheses supporting rapid resumption is implicit. If the content were already explicit, participants could respond right away, and there would be no need to wait and test against the display. In other words, within the present framework, perceptual hypotheses are operationalized as implicit, awaiting confirmation before they can generate the explicit response that leads to rapid resumption. The status of perceptual hypotheses as implicit captures important features of rapid resumption and motivates consideration of convergent findings from the literature on implicit visual learning.

\section{Similarities Between Rapid Resumption and Contextual Cuing}

If the same display is searched numerous times, it is likely that sometimes the very same perceptual hypoth- 
eses will be generated and tested. However, if perceptual hypotheses contain only distractor location information, functionally equivalent hypotheses may be generated by displays with equivalent spatial layouts. Repeatedly using the same perceptual hypothesis (e.g., searching a display that generates the same hypothesis) could result in a hypothesis template; that is, the content could be stored and activated later in a relevant context. An experimental test of this prediction already exists in the phenomenon of contextual cuing (Brockmole \& Henderson, 2006; Chun \& Jiang, 1998, 2003; Jiang \& Leung, 2005; Jiang et al., 2005; Peterson \& Kramer, 2001). Contextual cuing occurs over the course of a search experiment when participants are tested with a small subset of displays, using repeated spatial layouts, intermixed in a large set of displays with novel spatial layouts. The repeated layouts allow the locations of the distractors to predict the location of the target (but not its identity). Participants extract this predictive information implicitly and come to search through the repeated displays more quickly. As will be detailed below, prior work on contextual cuing lines up quite well with the present rapid resumption experiments.

Spatial extent. Rapid resumption operates over a local subset of about three to four search items. The original and nearly all subsequent spatial contextual cuing studies used entire search arrays as the repeated predictor. However, subsequent work revealed that the predictive context is more spatially local around the target (Olson $\&$ Chun, 2002). In fact, the effective input to contextual cuing can be restricted to three search items (Brady \& Chun, 2007) - specifically, the target and the nearest two distractors. Repeating only the two distractor items nearest to the target provided a benefit of the same magnitude as repeating an entire array of distractor locations. Thus, both contextual cuing and rapid resumption operate over a local subset of search items.

Featural resolution. The results of Experiments 1 and 2 suggest that perceptual search hypotheses contain coarse information about the location of distractors near the target, but not necessarily fine-grained featural details (in this case, distractor orientation). Likewise, contextual cuing built up from search with one set of distractors can transfer over to another set of distractors, demonstrating that distractor locations can be extracted and used independently of their feature detail. For example, Chun and Jiang (1998, Experiment 2) demonstrated that contextual cuing transferred across changes in the identities of distractors, when the locations of distractors and targets were constant (but see Jiang \& Song, 2005).

Task relevance. Visual search can be made more efficient in some cases by filtering out items that could not possibly be a target. There are numerous "low-level" features that facilitate such filtering (Egeth et al., 1972; Egeth et al., 1984; Treisman \& Gelade, 1980; Wolfe et al., 2003), and color and luminance are good examples. We find that perceptual hypotheses are formed selectively over items of the same task-relevant luminance as the target. Likewise, contextual cuing operates selectively over items of the same task-relevant color as the target (Jiang \& Chun, 2001; but see Jiang \& Leung, 2005).
Parallels between rapid resumption and contextual cuing suggest a general description of implicit processing in visual search. Resources underlying visual search are theorized to operate using iterative loops of information extraction and confirmation (hypothesis generation and testing). We have shown that by interrupting a processing loop, it is possible to study the effective content at a given point in time (at target detection), and this content is remarkably similar to the content that builds up over experience to produce contextual cuing. Although this position requires further empirical study to be fully asserted, the experiments presented here demonstrate promising convergence.

\section{Implicit to Explicit Processing in Visual Search}

Once a loop of extraction and confirmation passes its output to response selection, confirmed implicit perceptual hypotheses become the content of explicit consideration. Increased efficiency at any stage in this process can potentially produce RT benefits. Data exist at a temporal resolution bridging rapid resumption and contextual cuing, under the label priming of pop-out (Chun \& Nakayama, 2000; Malkovich \& Nakayama, 1994, 1996, 2000). Whereas rapid resumption operates within single search trials and contextual cuing builds up over many blocks of search trials, priming of pop-out is an effect within a single block of search (ranging up to about five to eight trials). In pop-out search, when the target is a feature singleton (e.g., a red target among yellow distractors), an RT benefit is observed from repeating a recently encountered target color or location. Considered in terms of perceptual hypotheses, this would be a case where a recently confirmed hypothesis benefits the efficiency of similar future hypotheses for a short range of time (Huang, Holcombe, \& Pashler, 2004). The alternative, purely speeding a response selection stage, was ruled out by the design of the original priming of pop-out experiments (Maljkovic \& Nakayama, 1994, 1996).

In conclusion, the present experiments suggest that sparse and useful preliminary evidence about targets and the layout of local, attended distractors accumulates and is usefully deployed prior to explicit target detection. Under a reentrant model, search processing operates in successive loops of extraction and confirmation. Information extracted prior to confirmation is very similar to the content that supports implicit learning of predictive, invariant information during visual search, as demonstrated in contextual cuing and priming of pop-out tasks. In visual processing, it is critical to transform implicit information into explicit information or behavior, and resuming interrupted search taps into this transformation in novel ways. Thus, the present results provide a converging link between diverse demonstrations of implicit and explicit processing in visual search.

\section{AUTHOR NOTE}

Correspondence concerning this article should be addressed to J. A. Jungé, Center for Cognitive Studies, Tufts University, 11 Miner Hall, Medford, MA 02155 (e-mail: justinjunge@gmail.com).

Note-Accepted by the previous editorial team, when Thomas H. Carr was Editor. 


\section{REFERENCES}

Alvarez, G. A., \& Cavanagh, P. (2004). The capacity of visual shortterm memory is set both by visual information load and by number of objects. Psychological Science, 15, 106-111.

BeCKer, M. W., \& PAshler, H. (2002). Volatile visual representations: Failing to detect changes in recently processed information. Psychonomic Bulletin \& Review, 9, 744-750.

Brady, T. F., \& Chun, M. M. (2007). Spatial constraints on learning in visual search: Modeling contextual cueing. Journal of Experimental Psychology: Human Perception \& Performance, 33, 798-815.

Brainard, D. H. (1997). The Psychophysics Toolbox. Spatial Vision, 10, 433-436.

Brockmole, J. R., \& Henderson, J. M. (2006). Using real-world scenes as contextual cues for search. Visual Cognition, 13, 99-108.

Chun, M. M., \& JiAng, Y. (1998). Contextual cueing: Implicit learning and memory of visual context guides spatial attention. Cognitive Psychology, 36, 28-71.

Chun, M. M., \& Jiang, Y. (2003). Implicit, long-term spatial context memory. Journal of Experimental Psychology: Learning, Memory, \& Cognition, 29, 224-234.

Chun, M. M., \& Nakayama, K. (2000). On the functional role of implicit visual memory for the adaptive deployment of attention across scenes. Visual Cognition, 7, 65-81.

Dennett, D. C. (1991). Consciousness explained. Boston: Little, Brown.

Di Lollo, V., Enns, J. T., \& Rensink, R. A. (2000). Competition for consciousness among visual events: The psychophysics of reentrant processing. Journal of Experimental Psychology: General, 129, 481-507.

Duncan, J., \& Humphreys, G. W. (1989). Visual search and stimulus similarity. Psychological Review, 96, 433-458.

Egeth, H. E., Jonides, J., \& Wall, S. (1972). Parallel processing of multielement displays. Cognitive Psychology, 3, 674-698.

Egeth, H. E., Virzi, R. A., \& Garbart, H. (1984). Searching for conjunctively defined targets. Journal of Experimental Psychology: Human Perception \& Performance, 10, 32-39.

HAN, S.-H., \& KIM, M.-S. (2004). Visual search does not remain efficient when executive working memory is working. Psychological Science, 15, 623-628.

Huang, L., Holcombe, A. O., \& Pashler, H. (2004). Repetition priming in visual search: Episodic retrieval, not feature priming. Memory \& Cognition, 32, 12-20.

JiAng, Y., \& CHUN, M. M. (2001). Selective attention modulates implicit learning. Quarterly Journal of Experimental Psychology, 54A, 1105-1124.

JIANG, Y., \& LEUNG, A. W. (2005). Implicit learning of ignored visual context. Psychonomic Bulletin \& Review, 12, 100-106.

JiANG, Y., OlsON, I. R., \& CHUN, M. M. (2000). Organization of visual short-term memory. Journal of Experimental Psychology: Learning, Memory, \& Cognition, 26, 683-702.

JiANG, Y., \& Song, J.-H. (2005). Hyperspecificity in visual learning: Learning of spatial layout is contingent on item identity. Journal of Experimental Psychology: Human Perception \& Performance, 31, 1439-1448.

Jiang, Y., Song, J.-H., \& Rigas, A. (2005). High-capacity spatial contextual memory. Psychonomic Bulletin \& Review, 12, 524-529.

KRISTJÁnNSON, A. (2000). In search of remembrance: Evidence for memory in visual search. Psychological Science, 11, 328-332.

Lleras, A., Rensink, R. A., \& EnNs, J. T. (2004). Rapid resumption is modulated by high-level strategies [Abstract]. Journal of Vision, 4, 180a. Available at journalofvision.org/4/8/180/. doi:10.1167/4.8.180

Lleras, A., Rensink, R. A., \& Enns, J. T. (2005). Rapid resumption of interrupted visual search: New insights on the interaction between vision and memory. Psychological Science, 16, 684-688.
Lleras, A., Rensink, R. A., \& Enns, J. T. (2007). Consequences of display changes during interrupted visual search: Rapid resumption is target specific. Perception \& Psychophysics, 69, 980-993.

MaljKovic, V., \& Nakayama, K. (1994). Priming of pop-out: I. Role of features. Memory \& Cognition, 22, 657-672.

Maljkovic, V., \& Nakayama, K. (1996). Priming of pop-out: II. The role of position. Perception \& Psychophysics, 58, 977-991.

MaljKovic, V., \& Nakayama, K. (2000). Priming of popout: III. A short-term implicit memory system beneficial for rapid target selection. Visual Cognition, 7, 571-595.

OH, S.-H., \& KIM, M.-S. (2004). The role of spatial working memory in visual search efficiency. Psychonomic Bulletin \& Review, 11, 275-281.

Olson, I. R., \& Chun, M. M. (2002). Perceptual constraints on implicit learning of spatial context. Visual Cognition, 9, 273-302.

Peterson, M. S., \& Kramer, A. F. (2001). Attentional guidance of the eyes by contextual information and abrupt onsets. Perception \& Psychophysics, 63, 1239-1249.

Peterson, M. S., Kramer, A. F., Wang, R. F., Irwin, D. E., \& McCarley, J. S. (2001). Visual search has memory. Psychological Science, 12, 287-292.

Rauschenberger, R., \& Yantis, S. (2006). Perceptual encoding efficiency in visual search. Journal of Experimental Psychology: General, 135, 116-131.

RENSINK, R. A. (2000). The dynamic representation of scenes. Visual Cognition, 7, 17-42.

Shore, D. I., \& Klein, R. M. (2000). On the manifestations of memory in visual search. Spatial Vision, 14, 59-75.

Treisman, A. M., \& Gelade, G. (1980). A feature-integration theory of attention. Cognitive Psychology, 12, 97-136.

Vogel, E. K., Woodman, G. F., \& Luck, S. J. (2006). The time course of consolidation in visual working memory. Journal of Experimental Psychology: Human Perception \& Performance, 32, 1436-1451.

Wolfe, J. M. (1994). Guided Search 2.0: A revised model of visual search. Psychonomic Bulletin \& Review, 1, 202-238.

Wolfe, J. M. (1998). What can 1 million trials tell us about visual search? Psychological Science, 9, 33-39.

Wolfe, J. M., Butcher, S. J., Lee, C., \& Hyle, M. (2003). Changing your mind: On the contributions of top-down and bottom-up guidance in visual search for feature singletons. Journal of Experimental Psychology: Human Perception \& Performance, 29, 483-502.

Woodman, G. F., \& Chun, M. M. (2006). The role of working memory and long-term memory in visual search. Visual Cognition, 14, 808-830.

Woodman, G. F., \& Luck, S. J. (2004). Visual search is slowed when visuospatial working memory is occupied. Psychonomic Bulletin \& Review, 11, 269-274.

Woodman, G. F., Vogel, E. K., \& Luck, S. J. (2001). Visual search remains efficient when visual working memory is full. Psychological Science, 12, 219-224.

\section{NOTE}

1. These manipulations provide an important control for interpreting Experiments 1 and $2 \mathrm{~A}$. A deflationary account of the differing results observed in these two experiments would be that the total number of pixels altered is greater when distractor locations change than when their orientations change, and, thus, it is this raw difference that drives the observed performance difference between experiments. However, if changing the location of distractors outside of a participant's attentional set fails to interfere with rapid resumption, this deflationary account can be dismissed, since total pixels changed is equated.

(Manuscript received September 26, 2006; revision accepted for publication November 24, 2008.) 